\title{
Congenital lobar emphysema in bilateral lung lobes: a case report
}

\author{
Qiaoling Lei, Wen Zeng, Rong Ju \\ Department of Neonatology, Chengdu Women's and Children's Central Hospital, Chengdu, China \\ Correspondence to: Rong Ju. Department of Neonatology, Chengdu Women's and Children's Central Hospital, School of Medicine, University of \\ Electronic Science and Technology of China, 1617 Riyue Avenue, Qingyang District, Chengdu 611731, China. Email: 330202352@qq.com.
}

\begin{abstract}
Congenital lobar emphysema (CLE) is a rare congenital anomaly of lung. It presents different respiratory symptoms due to affected lobar emphysema, compression atelectasis and mediastinal shift. It can affect one or more lobes. There is usually no typical clinical manifestation in clinic. Typical X-ray can help to diagnose. We report a case of neonatal CLE, which first appeared in the right middle lobar. After right middle lobe (RML) lobectomy, respiratory distress appeared again. Emphysema was found again in the left lower lung. The multiple lobes emphysema appear at different times, and this case occurred after lobectomy.
\end{abstract}

Keywords: Congenital lobar emphysema (CLE); lobectomy; hyperinflation; atelectasis; case report

Submitted Nov 27, 2019. Accepted for publication May 11, 2020.

doi: $10.21037 / \mathrm{tp}-19-147$

View this article at: http://dx.doi.org/10.21037/tp-19-147

\section{Introduction}

Congenital lobar emphysema (CLE) is a rare developmental abnormality of the lung with hyperinflation of one or more lobes, secondary atelectasis of other lobes and mediastinal displacement (1). CLE may present in different manifestations, from neonatal acute respiratory distress to recurrent dyspnea or pulmonary infection in children. It can be diagnosed at any time, including the prenatal, neonatal, infant, and adult periods, particularly during the neonatal period (2). Early recognition of CLE and early surgical intervention are life-saving. Despite the progress in the diagnostic techniques, the diagnosis and management of CLE are still relatively difficult, and CLE may be misdiagnosed as pneumonia or tension pneumothorax (3).

Herein, we report a case of a neonatal CLE patient aged $10 \mathrm{~d}$ who presented with severe respiratory distress. The chest $\mathrm{X}$-ray were similar to those of neonatal pneumonia. The patient was initially diagnosed with neonatal pneumonia and respiratory failure. But the respiratory distress was non-responsive to invasive ventilation and anti-infective treatment. Subsequent serial radiographic evaluation showed CLE which first appeared in the right middle lobe (RML). The patient underwent RML lobectomy. The general condition improved postoperatively. However, the patient developed respiratory distress 5 days after surgery. Serial radiographic evaluation showed progressive air trapping and cyst formation in the left lower lobe necessitating a subtotal pneumonectomy. This is a bilateral, multilobed CLE, and multiple lobes appear one after another. The contralateral lobe emphysema occur after the pressure is relieved after operation. From this case, we learned multiple lobar emphysema can occur after partial lobectomy. We did not decompress the RML during the operation, and then explore the condition of the contralateral lobes, resulting in the emphysema of the contralateral lobes after the operation. Therefore, it is necessary to check the anatomical structures, external pressure to the airway and explore the condition of the contralateral lobes during the operation. We present the following case in accordance with the CARE reporting checklist (available at: http://dx.doi. org/10.21037/tp-19-147).

\section{Case presentation}

Informed written consent was obtained from his parents. A neonatal patient aged $10 \mathrm{~d}$, with a history of shortness of breath, wheezing, fever, and progressive tachypnea (respiratory rate $>60$ breaths/min) for $4 \mathrm{~d}$, was transferred to Chengdu Women's and Children's Central Hospital. After contact with a cold family members, the patient developed fever, tachypnea, spitting clinical manifestations 


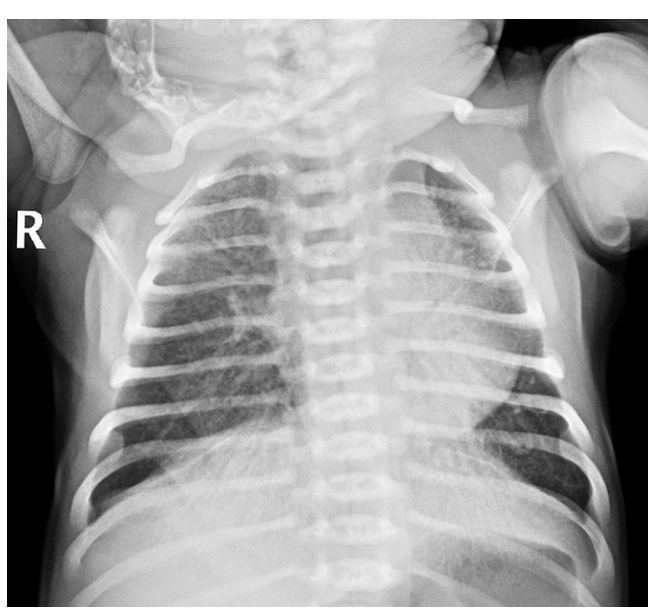

Figure 1 Chest X-ray shows slightly increased pulmonary texture as well as speckled and flocculent shadow in both lungs.

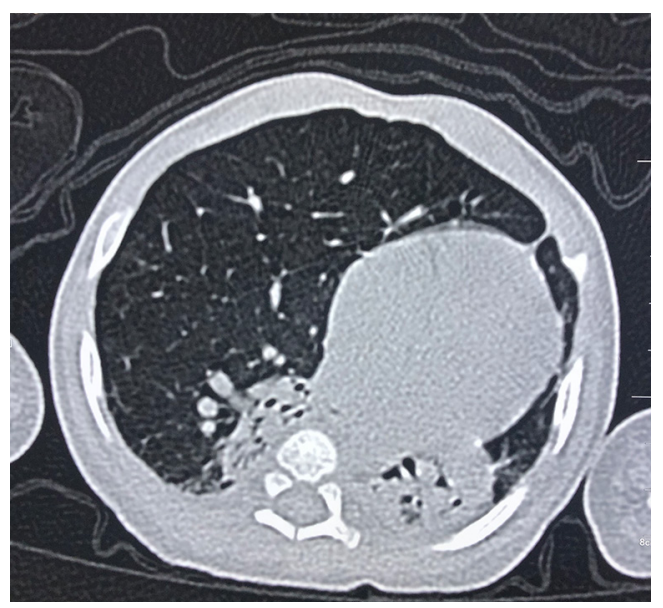

Figure 2 Chest CT shows hyperinflation of the RML, with pulmonary atelectasis (right-upper lobe, lower lobe, and left lung) and mediastinal displacement to the left. RML, right middle lobe.

on Day 6 after birth, with a maximum temperature of 38.2 degrees Celsius, and was admitted to a community hospital and initially diagnosed as neonatal pneumonia. He was given Budesonide aerosol inhalation, suction, Cefathiamidine anti-infection treatment. After 4 days of treatment, the child's temperature was normal. However, the respiratory distress was non-responsive to treatment and even aggravated despite of non-invasive ventilation. The patient was then transferred to our hospital on Day 10. This neonate was the second child from a physiological pregnancy and born at term via vaginal delivery with the birth weight of $3,750 \mathrm{~g}$. No asphyxia was reported at birth. The antenatal history of the mother was uneventful. His parents were not consanguineous and there was no family history of neonatal death in the first- and second-degree relatives.

Physical examination on admission showed the body temperature was $36.7^{\circ} \mathrm{C}$, the pulse was 145 beats $/ \mathrm{min}$, the respiratory rate was 66 breaths/min, the blood pressure was $65 / 36 \mathrm{mmHg}$ and the transcutaneous oxygen saturation was $78 \%$ (on oxygen inhalation). In addition, cyanosis, dyspnea, nares flaring, mild intercostal and subcostal retractions, and coarse bilateral breath sounds on auscultation were also found on physical examination. Cardiac examination showed regular rate and rhythm, normal S1 and S2, no murmurs, and normal pulses. The neonate was immediately intubated and ventilated (mode: high-frequency oscillatory ventilation, $\mathrm{FiO}_{2}$ at $60 \%, \mathrm{f} 11 \mathrm{HZ}, \mathrm{MAP}$ at $17 \mathrm{cmH}_{2} \mathrm{O}, \triangle \mathrm{P}$ at $31 \mathrm{cmH}_{2} \mathrm{O}, \mathrm{SPO}_{2}$ at $93 \%$ ). Blood gas analysis showed the $\mathrm{pH}$ was 7.357 , the $\mathrm{PO}_{2}$ was $50.4 \mathrm{mmHg}$, the $\mathrm{PCO}_{2}$ was $41.4 \mathrm{mmHg}$ and the $\mathrm{BE}$ was $-2.3 \mathrm{mmol} / \mathrm{L}$. The findings on chest $\mathrm{X}$-ray were similar to those of neonatal pneumonia (Figure 1). Echocardiography revealed a patent foramen ovale (left-to-right shunt) and normal left ventricular systolic function. The patient was diagnosed with neonatal pneumonia and respiratory failure. Ventilation, antiinfection (cefoperazone schubatam), sedation, and other treatments were administered.

After treatment for $6 \mathrm{~d}$, the dyspnea in this patient showed no improvement. Then, the antibiotics were replaced with meropenem. Persistent dyspnea and wheezing were observed as the disease progressed; thereafter, chest CT scanning was conducted on Day 30 and showed the volumes of the right upper lobe, lower lobe, and left lung decreased, the RML markedly expanded, and hyperlucent lung was observed, but the trachea and main bronchi were not obstructed. The patient received anti-infective, (meropenem), midazolam sedative, aminophylline, procaterol spasmolytic, and antiasthmatic treatments in the presence of mechanical ventilation (mode: synchronized intermittent mandatory ventilation, $\mathrm{FiO}_{2}$ at $35 \%$, RR, 30 beats/min, PIP at $21 \mathrm{cmH}_{2} \mathrm{O}$, PEEP at $5 \mathrm{cmH}_{2} \mathrm{O}$, iT at $0.5 \mathrm{~s}$, and $\mathrm{SPO}_{2}$ at $95 \%$ ). As the symptoms persisted, and wheezing recurred on Day 77. A second chest CT scanning was done due to suspicion of CLE, and pneumonia, hyperinflation of the RML, compression atelectasis of the right and left lobes, and mediastinal hernia (Figure 2) were noted. As compared to the findings on prior CT, these suggested the pulmonary emphysema in the RML worsened. To differentiate it from other lung diseases, further fiberoptic bronchoscopy was performed. Bronchoscopic examination 
revealed endobronchial inflammation without other pathological changes. CLE of the RML was then diagnosed based on the imaging findings.

The patient underwent RML lobectomy and right thoracic drainage on Day 79. During the thoracotomy, severe hyperinflation of the RML was found and characterized by the white lung and loss of elasticity (Figure 3). The compressed upper and lower lobes of the right lung showed atelectasis and consolidation. The histopathology of the RML indicated most of the terminal bronchioles and alveoli were dilated; some alveolar septa ruptured and fused to form bullae. The general condition improved postoperatively. However, the patient developed respiratory distress 5 days

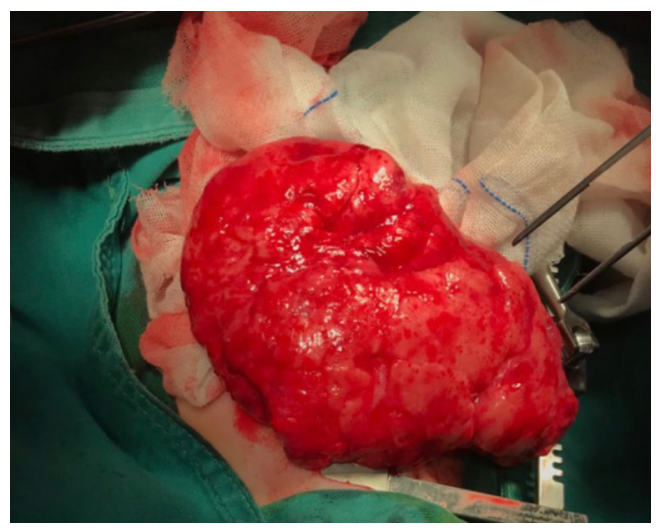

Figure 3 Lung tissues from the RML collected during the surgery. $\mathrm{RML}$, right middle lobe. after surgery. Chest X-ray and CT revealed the hyperinflation of the left lower lobe, causing the mediastinal displacement to the right and the compression of the remaining lung (Figure 4). Thus, the emphysema in the lower left lobe after the RML lobectomy was diagnosed. A second surgery was proposed, but the parents opted for conservative treatment. He died at 99 days and didn't complete the genetic test (Figure 5).

\section{Discussion}

CLE is a rare disease of the lung with an incidence of about 1 per 20,000 to 30,000 births (4). Low incidence and co-infection often cause misdiagnosis and delayed treatment. The left upper lobe and the middle lobe are the most commonly affected lobes, followed by the right upper lobe and the right lower lobe, and CLE affecting two ipsilateral lobes is extremely rare. The etiology of $50 \%$ CLE remains unclear (5). Congenital cartilage defect accounts for $1 / 4$ of the causes and is also the most common cause (2). It is presumed that the bronchial cartilage defect may lead to excessive inflation and air trapping on expiration due to the ball-valve effect. This causes lung lobar hyperinflation (5). The clinical manifestations are mainly respiratory dysfunction and recurrent pulmonary infection. The age at the onset of respiratory symptoms varies considerably, ranging from neonatal period to adulthood. Respiratory dysfunction is predominant within 6 months after birth; however,
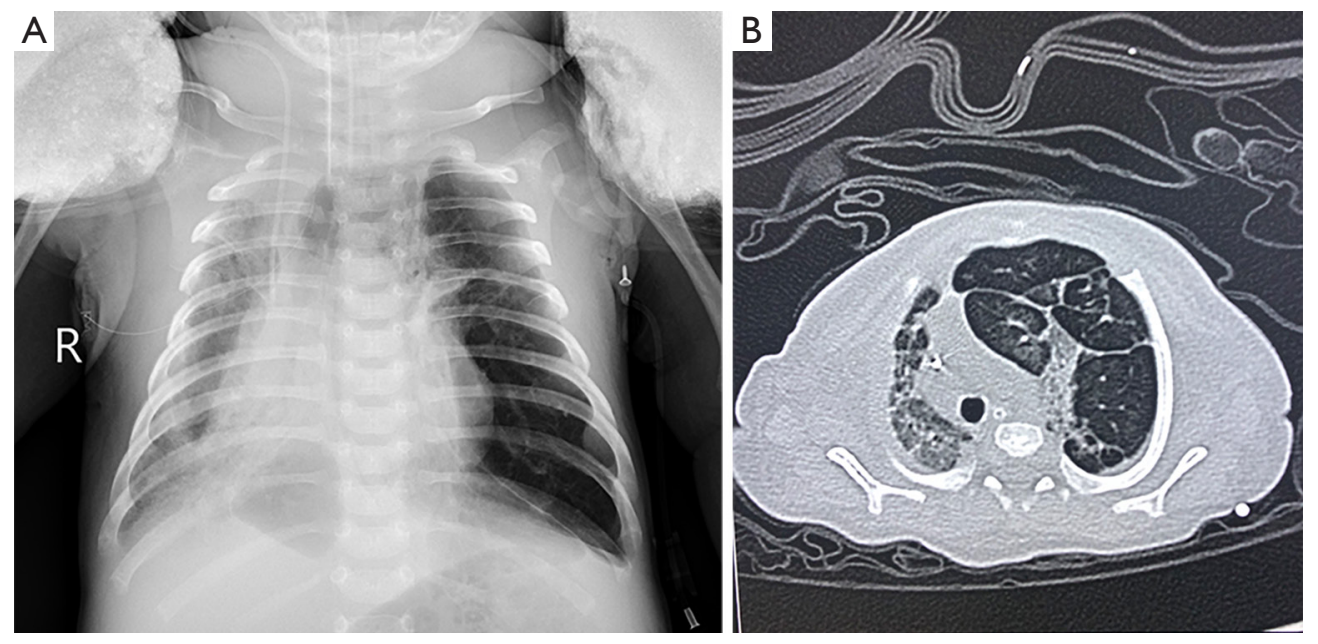

Figure 4 Chest X-ray (9 days after surgery) (A) and CT (11 days after surgery) (B) show hyperlucency in the left lower lobe, with pulmonary atelectasis and mediastinal displacement to the left. 


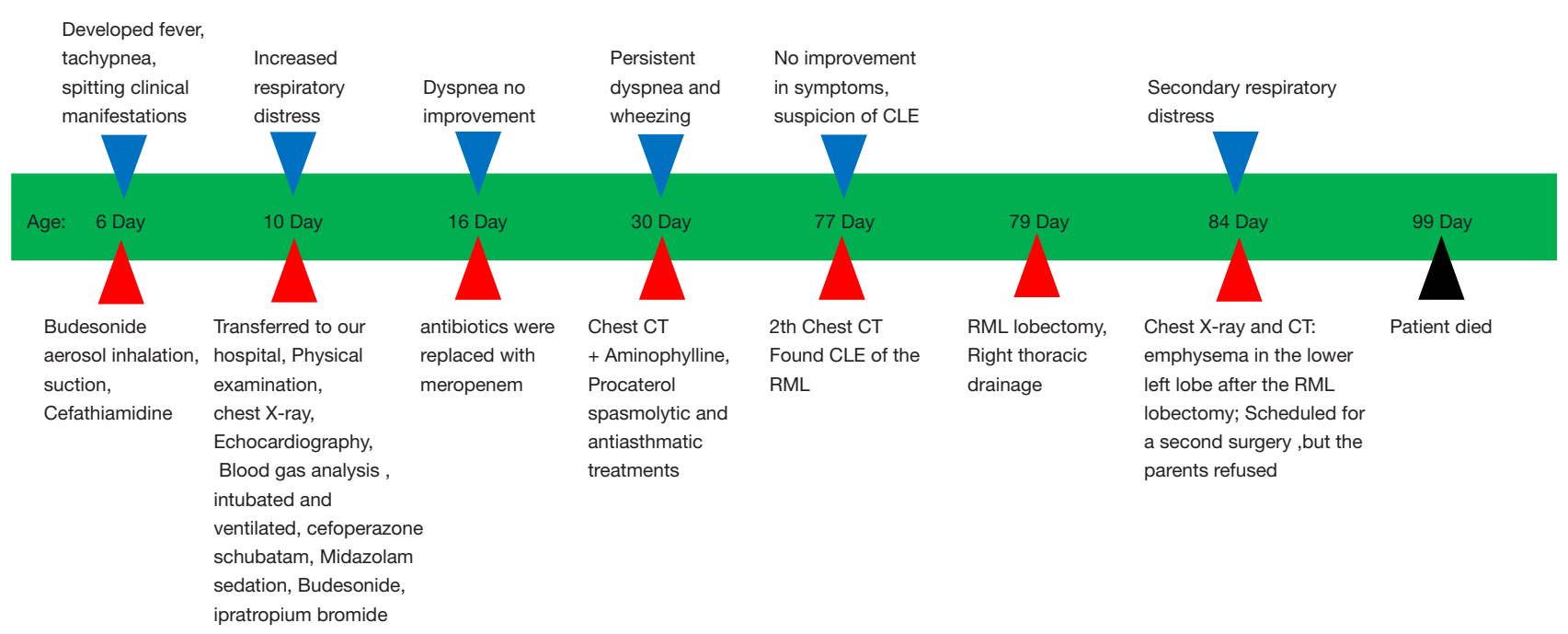

Figure 5 Timeline of this case.

it is also reported in patients older than 6 months (6). Adult patient may be asymptomatic (7) and are often diagnosed with CLE incidentally. Recurrent pulmonary infection is the most common manifestation in children and adults. CLE usually has no specific clinical manifestations, which poses diagnostic and therapeutic dilemmas (8). In clinical practice, it should be differentiated from many other lung diseases. Prenatal ultrasonography (1) and magnetic resonance imaging can be used to differentiate CLE from other congenital pulmonary lesions in the uterus at an early stage, such as bronchopulmonary sequestration, congenital cystic adenomatoid malformation, congenital diaphragmatic hernia, and bronchogenic cyst (9). Some studies have reported that chest X-ray facilitates the diagnosis of CLE (2). The CLE on chest X-ray presents hyperinflation of the affected lobe with the compression of normal lung parenchyma and the displacement of contralateral mediastinum. Regardless, it is often confused with compensatory emphysema caused by atelectasis or pneumothorax based on clinical presentations, which affects its diagnosis and then delays its treatment in the early stage. Chest CT is important for confirmed diagnosis $(2,10)$. The flexible bronchoscopy can be used to evaluate for alternative causes of lung hyperinflation including vascular compression, foreign bodies, inspissated mucus, airway anatomical anomalies and endobronchial tumors. Bronchoscopy also can be used to exclude foreign body aspiration and identify candidates for conservative treatment. The resection of the affected lung lobe is recommended for children with severe respiratory symptoms. Surgery is also safe for patients with cardiac dysfunction (11). The earlier the presentation is, the greater is the need for surgery (7). The mortality is low in children with CLE undergoing surgery (11). Asymptomatic patients or patients with mild respiratory symptoms in older children should be closely monitored and receive conservative treatment (2). Eigen et al. found that lung growth and function in a small group of children with CLE treated conservatively were no different from that of children treated surgically by lobectomy (12).

The patient in our report was initially misdiagnosed as pneumonia based on symptoms of respiratory distress and chest $\mathrm{X}$-ray. As the disease progressed, the diagnosis was confirmed only by typical findings on chest CT. After lobectomy, the symptoms deteriorated again at 5 days after surgery and CLE appeared again in the contralateral lung (left lower lobe), as determined by chest X-ray and chest CT. In available studies (13), patients with CLE involving multiple lobes were rarely reported. Olutoye et al. (9) reported that the emphysema was found in the fetus. RML lobectomy was performed three days after birth. Postoperatively, it was hard for the child to wean the ventilator. Progressive air trapping and cyst formation in the remaining right lung. The emphysema occurs again in the ipsilateral residual lobe after RML lobectomy. Subtotal pneumonectomy, except for the superior segment of the right upper lobe, was conducted. Different from the case we reported, their RML resection was performed in the early postnatal period, and the multiple lobes were all ipsilateral lobes. RML lobectomy was performed 79 days after birth 
in our case. The emphysema occurred on the contralateral lobes. Therefore, we speculate that the time of occurrence of multiple lobes in succession has nothing to do with the time of operation and ipsilateral or contralateral lobes. Perea (14) reported 4 cases of bilateral CLE. The RML and left upper lobar emphysema were found to be involved by CTA before lobectomy. Two patients underwent RML lobectomy and one patient underwent the left upper lobe lobectomy. The three patients remained stable during follow-up and did not need further surgery. But, one patient underwent left upper lobectomy. In the course of postoperative, there was re-expansion of the RML, which led to the decision of RML lobectomy. What's different in our case is that the bilaterally lesion lobes were found after RML lobectomy, and respiratory distress occurred after operation, so it needs to be operated again. We speculated that this might be attributed to the large volume of the affected lobe, compressing the ipsilateral and contralateral lobes. After RML lobectomy, pressure from the right side decreased, leading to increased ventilation in the left lower lobe, but the left lower lobe was also a diseased lobe, leading to hyperinflation presenting as emphysema. The specific mechanism should be further elucidated. We didn't expect that there would be other emphysema after the operation, and we didn't communicate with the parents about this. When other lobe emphysema occurs again after the operation, the parents choose to refuse to the second operation. Whether emphysema would occur in other lobes after second surgery was uncertain. Therefore, we should evaluate the postoperative recurrence of lobar emphysema before operation. But not all multiple lobes CLE need to be subsequent lobectomy. There is evidence that $75 \%$ of patients do not need, which may indicate that staged surgery is more beneficial than single-stage surgery, and it can be seen whether further surgery is needed. Multiple lobar emphysema may occur after partial lobectomy. How to avoid postoperative emphysema of other lobes? There are two things we haven't done well in our case. First, Perea (14) reminds that external compression is one of the causes of the disease during surgery, the surgeon should check the anatomical structures and make sure that there is no variation in the anatomy of the airway or external pressure to the airway. In our case, we did not explore the anatomical structure of the contralateral lung during the operation. Secondly, endobronchial obstruction techniques can be used to assess the potential impact of lobectomy when the etiology of hyperinflation is unclear because of complex respiratory comorbidities (14). In this case, we can perform endobronchial occlusion RML before operation, and then observe the relaxations of other lung lobes and the parameters of ventilator.

In conclusion, our findings indicate emphysema may potentially recur in other lobes after surgery for CLE. Therefore, serial radiographic evaluation is needed and patients should be closely monitored preoperatively and postoperatively. It is necessary for us to assess further physiologic of residual lung lobe before operation, and explore the anatomical structure of the contralateral lung during the operation.

\section{Acknowledgments}

Funding: None.

\section{Footnote}

Reporting Checklist: The authors have completed the CARE reporting checklist. Available at http://dx.doi.org/10.21037/ tp-19-147

Conflicts of Interest: All authors have completed the ICMJE uniform disclosure form (available at: http://dx.doi. org/10.21037/tp-19-147). The authors have no conflicts of interest to declare.

Ethical Statement: The authors are accountable for all aspects of the work in ensuring that questions related to the accuracy or integrity of any part of the work are appropriately investigated and resolved. Consent was obtained from relatives of the patient for publication of this report and any accompanying images.

Open Access Statement: This is an Open Access article distributed in accordance with the Creative Commons Attribution-NonCommercial-NoDerivs 4.0 International License (CC BY-NC-ND 4.0), which permits the noncommercial replication and distribution of the article with the strict proviso that no changes or edits are made and the original work is properly cited (including links to both the formal publication through the relevant DOI and the license). See: https://creativecommons.org/licenses/by-nc-nd/4.0/.

\section{References}

1. Prabhu M, Joseph TT. Congenital lobar emphysema: Challenges in diagnosis and ventilation. Anesth Essays Res 
2012;6:203-6.

2. Karnak I, Senocak ME, Ciftci AO, et al. Congenital lobar emphysema: diagnostic and therapeutic considerations. J Pediatr Surg 1999;34:1347-51.

3. Tempe DK, Virmani S, Javetkar S, et al. Congenital lobar emphysema: pitfalls and management. Ann Card Anaesth 2010;13:53-8.

4. Pariente G, Aviram M, Landau D, et al. Prenatal diagnosis of congenital lobar emphysema: case report and review of the literature. J Ultrasound Med 2009;28:1081-4.

5. Chinya A, Pandey PR, Sinha SK, et al. Congenital lobar emphysema: Pitfalls in diagnosis. Lung India 2016;33:317-9.

6. Man DW, Hamdy MH, Hendry GM, et al. Congenital lobar emphysema: problems in diagnosis and management. Arch Dis Child 1983;58:709-12.

7. von Ranke FM, Freitas HMP, Dinoá V, et al. Congenital lobar emphysema. Radiol Bras 2018;51:205-6.

8. Saini S, Prakash S, Rajeev M, et al. Congenital Lobar Emphysema: Anaesthetic Challenges and Review of

Cite this article as: Lei Q, Zeng W, Ju R. Congenital lobar emphysema in bilateral lung lobes: a case report. Transl Pediatr 2020;9(3):256-261. doi: 10.21037/tp-19-147
Literature. J Clin Diagn Res 2017;11:UD04-UD06.

9. Olutoye OO, Coleman BG, Hubbard AM, et al. Prenatal diagnosis and management of congenital lobar emphysema. J Pediatr Surg 2000;35:792-5.

10. Thakral CL, Maji DC, Sajwani MJ. Congenital lobar emphysema: experience with 21 cases. Pediatr Surg Int 2001;17:88-91.

11. Andrade CF, Ferreira HP, Fischer GB. Congenital lung malformations. J Bras Pneumol 2011;37:259-71.

12. Eigen H, Lemen RJ, Waring WW. Congenital lobar emphysema: long-term evaluation of surgically and conservatively treated children. Am Rev Respir Dis 1976;113:823-31.

13. Abushahin AM, Tuffaha AS, Khalil NK, et al. Bilateral congenital lobar emphysema: A rare cause for respiratory distress in infancy. Ann Thorac Med 2012;7:250-2.

14. Perea L, Blinman T, Piccione J, et al. Bilateral congenital lobar emphysema: staged management. J Pediatr Surg 2017;52:1442-5. 